Fizika Nizkikh Temperatur, 1999, v. 25, No 8/9, p. 820-823

\title{
De Haas-van Alphen effect and superconductivity
}

\author{
Lev P. Gor'kov \\ National High Magnetic Field Laboratory, Florida State University, \\ 1800 E. Paul Dirac Dr., Tallahassee, FL 32310 \\ L. D. Landau Institute for Theoretical Physics, Russian Academy of Sciences, 117334 Moscow, Russia \\ E-mail: gorkov@magnet.fsu.edu \\ Received March 17, 1999
}

\begin{abstract}
The problem of the robust de Haas-van Alphen oscillations seen experimentally in mixed superconducting state is discussed. The new threshold mechanism is suggested to explain persistence of the quantum oscillations deep in the superconducting state.

PACS: 74.20.Fg, 72.15.Gd, 74.60.-w
\end{abstract}

I am very glad to be able to provide a paper for the issue dedicated to the memory of Professor B. I. Verkin. His own contributions and his efforts to promote condensed matter research in Kharkov to new highs were, indeed, impressive and successful. Currently, the Institute for Physics and Technology at Low Temperatures (FTINT) which he had helped to organize from scratch enjoys a reputation of one of the main scientific centers in the field.

The Kharkov School is famous for its pioneering studies of normal metals' properties by various means and tools. Thus, among others, observations of quantum oscillations are the most direct assessment of electronic spectra in metals via the famous Lifshitz-Kosevich formula for the de Haas-van Alphen (dHvA) effect. Fermi surfaces (FS) of countless metals and intermetallic compounds have been determined and classified with the use of this method during the last flew decades.

It is worthwhile to emphasize that the very concept of FS is at the core of the Landau Fermiliquid (FL) theory. With the remarkable recent progress in synthesis of new materials, many of which reveal unexpected peculiarities in their physical properties, the question arose whether the Landau FL theory remains applicable in spite of the observed complications, or electron-electron interactions, being strong enough, may breach the FLtheory. Studies of the dHvA effect would provide the most direct test of the FL-assertions.

Below we address the issue of existence of the dHvA oscillations in the superconducting state. Whatever is the mechanism of interactions causing superconductivity, the latter may usually be well understood in terms of the BCS microscopic theory. The truth is that it is far from being clear whether the BCS scheme remains applicable, say, in cuprates, heavy fermions (HF), or borocarbides, to mention a few. However, the BCS theory is based upon the FL concept. If the dHvA-effect in superconducting state in these materials (see below) could be described in frameworks of the BCS scheme, it would become an indirect proof of FL for these substances.

The dHvA effect in normal metals is nothing but the WKB phenomena. An electron possessing large momentum, $p_{F} \sim a^{-1}$, its levels in magnetic field are equidistant near the chemical potential, $\mu$ ( $\mu \equiv E_{F}$, the Fermi energy):

$$
E_{N}=\omega_{c}\left(N+\frac{1}{2}\right)
$$

where $\omega_{c}$, the cyclotron frequency, is the characteristic of an effective electron mass in case of some arbitrary FS. A minor variation in the field, $B$, such that $\Delta B / B \sim \omega_{c} / E_{F}<<1$, may push a level across the chemical potential causing abrupt change in levels' occupation numbers and, hence, the step-like change in magnetization. (For simplicity we consider a two-dimensional (2D) case where this mechanism becomes most transparent). In the quasiclassical approximation an electron performs a closed-orbit motion (circular orbits, in an isotropic model), which may be equally well described as the Larmor motion in real space, or as the electron motion in the momentum representation along a 
closed orbit encircling a FS. Equation (1) is then nothing but the result of quantization in accordance with the Bohr correspondence principle.

The dHvA effect in superconducting state is observed in the second-type superconductors when the magnetic field is gradually decreased below the upper critical field, $H_{c 2}$. At $B<H_{c 2}$, the mixed state superconductivity [1] sets in. The magnetic field in the sample remains practically homogeneous, $B=\bar{B}$, while the superconducting order parameter periodically varies in space. At $B \ll H_{c 2}$ the gap structure corresponds to a lattice of vortices (intervortex distance, $d$, being larger than $\xi_{0}$, the coherence length which defines the vortex core size) separated by the «bulk» where the amplitude of the gap is saturated to a constant, $\Delta_{B}$ :

$$
\Delta(\mathbf{r}, \mathbf{p}) \simeq \Delta_{B}(\mathbf{p}) \exp \{i \varphi(\mathbf{r})\}
$$

$\left[\Delta_{B}(\mathbf{p})\right.$ depends on $\bar{B}$, although in order of magnitude it is close to the value of the gap in the absence of field].

A challenge, and a theoretical puzzle, is that the dHvA effect is observed in the well-developed superconducting state, down to $B \sim 0.2 H_{c 2}$. This fact apparently contradicts our intuitive notion of the dHvA effect resulting from the mechanism of crossing the chemical potential by an energy level. Indeed, although vortex cores produce some low energy excitation, these are known to be localized inside the core. The «bulk» of the superconductor, at distances $r \sim d>>\xi_{0}$ from the core is fully «gapped», according to Eq. (2). The chemical potential being positioned in the middle of the gap, levels on the two branches of superconducting excitations (even in the presence of the field, $\bar{B}$ ) would never cross the chemical potential, in particular, because

$$
\omega_{c} \ll \Delta, T_{c} .
$$

Equation (3) is a strong inequality, as follows from the microscopic theory, and may be rewritten in the form

$$
\frac{B}{H_{c 2}\left(p_{F} \xi_{0}\right)} \ll 1 .
$$

Therefore it is commonly accepted that well below $H_{c 2}$ an effective «Dingle» temperature

$$
T^{*} \sim \Delta
$$

would result in the fast decay of the dHvA signal. Experiments [2-4] strongly contradict the above arguments.
The field range $B \sim 0.2 H_{c 2}$ which has been reached experimentally in $[3,4]$ presents considerable difficulties for a theoretical analysis because the vortex lines are not yet well defined $\left(d \gtrsim \xi_{0}\right)$. However, it is natural to wonder about whether a specific and new mechanism lies behind the observed phenomenon of slow decay of the dHvA effect below $H_{c 2}$, and whether such a mechanism may be consistent with the microscopic theory. In [5-7] we have chosen to address the issue in the limit of:

$$
B<<H_{c 2} ; \quad \delta_{L}>d>>\xi_{0}
$$

to search for a new mechanism which may provide not so severe limitations on the dHvA amplitude, as is given by Eq. (5). Such mechanism does exist, indeed, and is explained below in some simple physical terms. A rigorous proof and the mathematical details can be found in [5-7].

At first, it is worth our while to discuss briefly a tempting guess that the persistence of the dHvA effect below $H_{c 2}$ may be caused by an unconventional symmetry of the superconducting order parameter. Once we consider a $2 D$ case (a cylindrical $\mathrm{FS}$ ), a natural suggestion is a « $d$-wave» pairing often assumed to be the ground state in high $T_{c}$ cuprates. In this model the gap disappears at the four points on the Fermi surface where the electronlike and the hole-like branches for excitations merge. It turned out [5], however, that although a level always exists in the presence of the magnetic field in the very vicinity of the chemical potential, its position is fixed. It is not changed by variations of the magnetic field. In other words, although the gapless « $d$-wave» superconductivity significantly changes the structure of levels, when compared to the «s-wave» pairing, the mechanism of crossing the chemical potential by a level at a variation of the magnetic field is again excluded.

The true mechanism which causes the dHvA oscillations in the superconducting state, at least in the limit $B \ll H_{c 2}$, Eq. (6), may be called the «threshold» mechanism (see $[6,7]$ ). There are two features in the superconducting energy spectrum which are responsible for its origin: an anisotropy of the superconducting order parameter, $\Delta(\mathbf{p})$, and the Doppler shift of the excitation energy by supercurrents flowing in the periodic vortex lattice, $\mathbf{v}_{s}(\mathbf{r})$. Consider them first in the absence of quantizing effects of the magnetic field.

If the gap, $\Delta(\mathbf{p})$, depends on the position of $\mathbf{p}$ along the Fermi surface, so that $\Delta_{\min }$ and $\Delta_{\max }$ are its minimal and maximum values, correspondingly, there are no excitations with the energy less than 
$\Delta_{\text {min }}$. Excitations with an energy $\varepsilon(\mathbf{p})>\Delta_{\max }$ may propagate freely to infinity in the real space. However, excitations with an energy such that $\Delta_{\min }<\varepsilon(\mathbf{p})<\Delta_{\max }$ can perform an infinite motion only if the momentum lies in the proper restricted angular cone.

Recall that excitations have no definite charges in the BCS-theory. Instead, there are two branches, one «electron-like» and one «hole-like». The meaning of these definitions is that the full electron charge is restored only far enough from the Fermi surface, while, say, the «electron-like» character of an excitation gradually diminishes by degrees as its energy tends to $\Delta_{\min }$.

An electron in the normal state placed in the magnetic field performs the motion along a closed orbit under the Lorentz force:

$$
\dot{\mathbf{p}}=\frac{e}{c}\left[\mathbf{v}_{F}(\mathbf{p}) \times \mathbf{B}\right] .
$$

Although Eq. (7) is not applicable for excitations in a superconductor since the excitation's charge is not fixed, as explained above, it helps to explain the origin of the «threshold» mechanism in a qualitative way. In the absence of the magnetic field excitations (the wave packets) move along straight lines, the momentum being preserved. The magnetic field bends the trajectories. While at an energy $\Delta_{\max }$ the excitation still can perform a closed motion (along the large Larmor-like orbit, $R_{L} \sim v_{F} / \omega_{c}$, in the real space, or along a trajectory encircling the Fermi surface, in the momentum space), this is not true for excitations with an energy less than $\Delta_{\max }$. Changing the direction of momentum the magnetic field brings it to the boundary of the angular cone beyond which extended motion of the wave packet is impossible. The «electron-like» character gets lost, and the excitation being rejected back (in the direction along FS), starts its motion as a «hole-like» particle. This process repeats on the other side of the allowed directions. Excitations with energies between $\Delta_{\text {min }}$ and $\Delta_{\max }$ will form «localized» states. Thus, there exists the energy threshold, $\varepsilon_{\text {th }}=\Delta_{\max }$, which separates the localized states and the «extended» states. For the latter an excitation behaves basically in the same way as ordinary electrons (except, of course, that their effective charge at $\varepsilon \geq \varepsilon_{\text {th }}$ is reduced from the bare electron charge and depends on energy). At the field variation the «extended» energy levels cross the threshold, becoming the «localized» ones.

The «threshold» effect caused by the anisotropy of the order parameter only, would lead to a signifi- cant dHvA effect even in the extreme case of Eq. (6) [5]:

$$
M_{\mathrm{osc}}^{s} \sim\left(\omega_{c} / \Delta\right)^{1 / 2} M_{\mathrm{osc}}^{N} .
$$

However, so far we have been neglecting the contribution from the Doppler effect. The Doppler shift in energy of excitations

$$
\varepsilon(\mathbf{p}) \rightarrow E(\mathbf{p})=\varepsilon(\mathbf{p})+\mathbf{p} \cdot \mathbf{v}_{s}(\mathbf{r})
$$

is due to the presence of the periodic supercurrents which flow even in the «bulk», i.e., away from the vortex cores. The second term in (9) is smaller than the scale of the gap, $\Delta_{\min } \sim \Delta_{\max }$. Its role is twofold. On the one side, the mechanism (9) may itself be the source of a threshold between «localized» and «extended» states. Indeed, consider a Larmor trajectory. Its radius, $R_{L} \sim v_{F} / \omega_{c}$, is much larger than $d$, the vortex lattice periodicity. Therefore, the quasiclassical packet may be thought in the first approximation as moving locally along a straight line, with some $\mathbf{p}$. Along that line $\mathbf{v}_{s}(\mathbf{r})$ varies but is limited in its value. Therefore, at $E<\Delta_{\max }+$ $+\left|\mathbf{p} \cdot \mathbf{v}_{s}(\mathbf{r})\right|_{\max }$, an excitation is bound again. Such a threshold effect exists even if the isotropic gap were chosen [7]

Unfortunately, the mechanism (9) also plays a destructive role on the dHvA effect. To understand this, recall that the gap anisotropy had led to the threshold position exactly at $\varepsilon=\Delta_{\max }$. The term $\mathbf{p} \cdot \mathbf{v}_{s}(\mathbf{r})$, although being small itself in the range of fields given by Eq. (6), significantly smears out the sharpness of this threshold due to spatial distribution of the superfluid velocity, $\mathbf{v}_{s}(\mathbf{r})$. As a result, the amplitude of the dHvA effect given by Eq. (8), suffers a sharp decrease, becoming again exponentially small. However, the value of an effective Dingle temperature turns out to be considerably reduced compared to Eq. (5):

$$
T_{\text {eff }}^{*} \sim \Delta\left(\xi_{0} / d\right) \ll \Delta .
$$

Using other language [5-7], the destructive effect of the Doppler shift may be interpreted as due to scattering of an electron moving along the Larmor orbit by the flux lines of the vortex lattice.

Thus, it is shown that, at least in the regime of Eq. (6), there exists a specific new mechanism of the dHvA oscillations in the developed superconducting mixed state that consists of crossing the threshold energy by levels of excitations in the magnetic field, while in the normal phase the oscillations originate from crossing the chemical potential. The regime of the magnetic field where $B \lesssim H_{c 2}$ is more difficult for theoretical analysis. 
However, we expect that similar threshold phenomena should take place even at $d \sim \xi_{0}$ to explain the robustness of the dHvA effect in the superconducting state.

The work was supported by the NHMFL through NSF cooperative agreement No. DMR-9016241 and the State of Florida.
1. A. A. Abrikosov, Zh. Eksp. Teor. Fiz. 32, 1442 (1957) [Sov. Phys. JETP 5, 1174 (1957)].

2. R. Corcoran, N. Harrison, C. J. Haworth, S. M. Hayden, P. Messon, M. Springford, and P. J. van der Wel, Physica B206-207, 534 (1995).

3. T. Terashima, C. Haworth, H. Takeya, S. Vji, H. Aoki, and K. Kadowaki, Phys. Rev. B56, 5120 (1997).

4. Y. Onuki, in: Proceedings of the Conference SCES'98, Paris (1998) (to be published).

5. L. P. Gor'kov and J. R. Schrieffer, Phys. Rev. Lett. 80, 3360 (1998).

6. L. P. Gor'kov and V. Z. Kresin, Pis'ma Zh. Eksp. Teor. Fiz. 67, 934 (1998) [JETP Lett. 67, 985 (1998)].

7. L. P. Gor'kov and J. R. Schrieffer, Cond-Mat $/ 9804328$ (1998) (to be published). 